\title{
Paper-based flexible metamaterial for microwave applications
}

\author{
Zidong Zhang ${ }^{1}$, Yaman Zhao ${ }^{2}$, Guohua Fan ${ }^{1}$, Wenjin Zhang ${ }^{1}$, Yao Liu ${ }^{1, *}$, Jiurong Liu $^{1}$, and Runhua Fan ${ }^{1,2, * *}$ \\ ${ }^{1}$ Key Laboratory for Liquid-Solid Structural Evolution and Processing of Materials (Ministry of Education), Shandong \\ University, Jinan 250061, PR China \\ ${ }^{2}$ College of Ocean Science and Engineering, Shanghai Maritime University, Shanghai 201306, PR China
}

Received: 10 November 2020 / Accepted: 21 December 2020

\begin{abstract}
Metamaterial has become a hotspot in many research fields, including electromagnetism, thermodynamics and mechanics, as it can offers additional design freedom for material to obtain novel properties. Especially for the electromagnetic devices, various interesting electromagnetic properties which cannot be found in nature materials can be realized, such as negative refraction, invisible cloak, etc. Herein, we provide an overview of paper-based metamaterial for microwave application. This work reviews the metamaterial realized on paper substrate, including the fabrication techniques, application fields, as well as the outlook on future directions of the paper-based metamaterial for the readership.
\end{abstract}

Keywords: Metamaterial / metasurface / microwave / paper / flexible

\section{Introduction}

Metamaterial usually are defined as a specially designed artificial structures to realize novel properties, such as negative refraction, inverse Doppler effect and backward Cherenkov radiations, which cannot be obtained in nature material [1-4]. Since the first demonstration of the negative refraction in microwave frequency via metamaterial by Smith et al. [5-7], the study of metamaterial grows rapidly and the concept of metamaterials has already been applied into many research fields, including optics [8], thermotic $[9,10]$, mechanics [11,12], acoustics [13-15], etc. Chen et al. focus on the topological phase of mechanical metamaterials which provide promising applications, such as vibration isolation, waveguiding without backscattering loss [16-19]. Valentine and co-workers experimentally verified the lefthand medium in the optical frequency range by using a fishnet structure [20]. Although metamaterials can be used to realize exotic properties and have a great potential in various applications, there still be several drawbacks need to be overcome, such as the complexity and high-cost of the fabrication process, lacking of tunability.

Recently, a great deal of attention has been paid to flexible metamaterials, in which flexible material has been used as the substrates [21-24]. Flexible substrates make it possible for metamaterials to be bendable, stretchable, and reversible, which is helpful to control the electromagnetic

\footnotetext{
* e-mail: liuyao@sdu.edu.cn

** e-mail: rhfan@shmtu.edu.cn
}

waves depending on specific requirements. Thus, flexible metamaterials could work on curved surface of various equipment or devices, not just flat surfaces. The flexibility brings a whole new range of applications to metamaterial, such as broadband microwave absorption, tunable electromagnetic resonance, wavefront manipulation on curved surface, wearable devices, etc. [25-28]. Choi et al. build I-shape resonators and dipole structure on spin-coated polyimide substrate for $\mathrm{THz}$ application [29]. Aksu et al. fabricated various type of plasmonic nanoantenna structure on different kind of flexible substrates using shadow mask lithography technique, including PDMS, parylene-C, and plastic food storage roll films based on low density polyethylene (LDPE) [30].

As mentioned above, there are different kinds of material can be used as substrate for flexible metamaterials fabrication [31], including PDMS, polyimide, and polymethylmethacrylate (PMMA), etc. [32-36]. Compare to the above polymer, paper has recently been considered as a highly promising substrate candidate for flexible metamaterials. As well known, paper is one of the most widely used materials in daily life, which is mass-producible, easyto-use, recyclable and disposable due to their biodegradability [37,38]. It has already been used for many interesting applications, including microfluidic mixing [39], biochemical reaction [40,41], etc. [42,43]

Moreover, the most attractive feature of paper is that it can be easily folded into different geometrical shape to meet various design considerations for different applications $[44,45]$, which making it an idea substrate candidate for the 
(a)
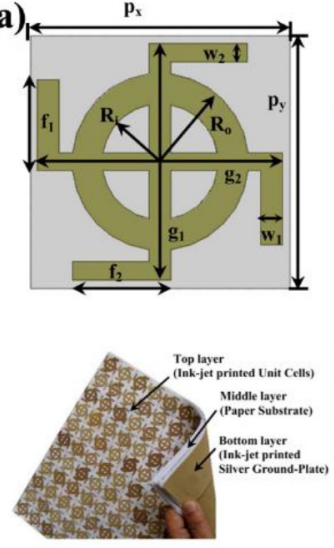
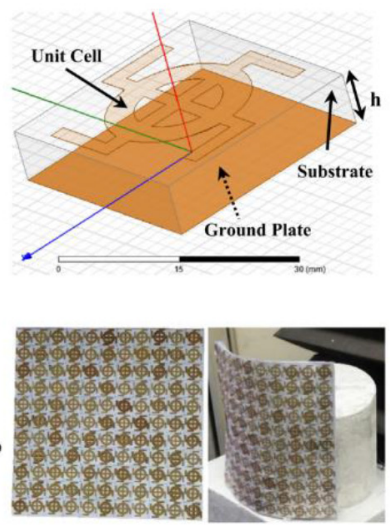

(b)

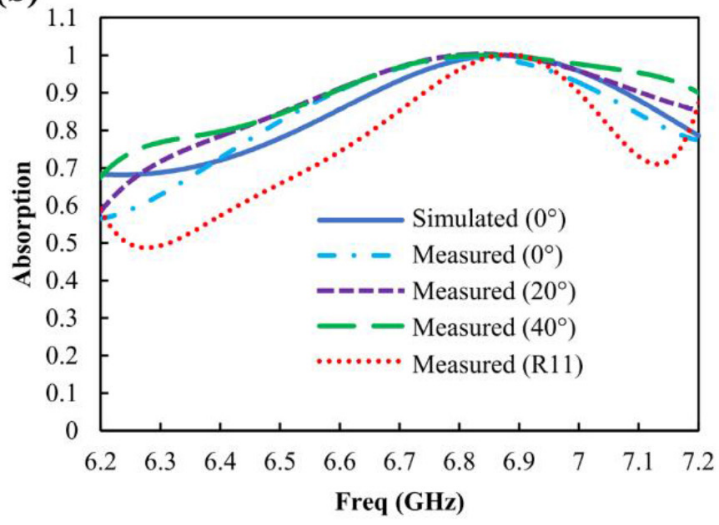

Fig. 1. (a) Schematic diagram and photograph of the ink-jet printing paper-based metamaterials. (b) Simulated and measured absorptivity at different incident angles on a planar surface, and at $0^{\circ}$ on a cylindrical surface (R11) [61]. Reproduced with permission from M. Momeni-Nasab et al., Int. J. Electron. Commun. 123, 153259 (2020). Copyright 2020 Elsevier. (This figure is subject to copyright protection and is not covered by a Creative Commons Commons license.)

fabrication of flexible metamaterials with tunable properties [46-51]. Meanwhile, it's demonstrated that the electromagnetic performance of metamaterials is independent on the various paper substrates. The abundant paper as raw materials is enough to ensure the mass production and application of this lightweight and versatile metamaterials, which also provides a new choice for waste paper recycling. Herein, we present a comprehensive review of the paper-based metamaterials, including the fabrication techniques, applications, as well as the future prospects. Both the advantages and the disadvantages of the paperbased metamaterial will be discussed in this review. The goal of this review is to outline these existed studies on paper-based metamaterial and then give an outlook of the future progress for the readership.

This review is organized as follows. Section 2 introduces the fabrication techniques of paper-based metamaterials and classifies the existed paper-based metamaterials. The applications of paper-based metamaterials are outlined in Section 3. In Section 4, we summarize the recent progress on this topic and discusses the future direction of paperbased metamaterials.

\section{Fabrication of paper-based metamaterial}

\subsection{Ink-jet printing}

Ink-jet printing method is one of the novel methods for paper-based metamaterials' fabrication [52-55]. Compare to the traditional fabrication methods, the ink-jet printing method has been given much attention due to its low-cost, timesaving, and eco-friendly. It has been widely used for the fabrication of paper-based metamaterial in $\mathrm{GHz}$ and $\mathrm{THz}$ frequency range [56-60].

Momeni-Nasab et al. design a flexible metamaterial absorber by using water-based reactive inks to from conductive patterns on paper substrate via ink-jet printing process [61]. As shown in Figure 1, the absorption levels of the metamaterial is higher than $99 \%$ at $6.81-6.84 \mathrm{GHz}$ frequency range. Moreover, it also shows angular insensitivity.
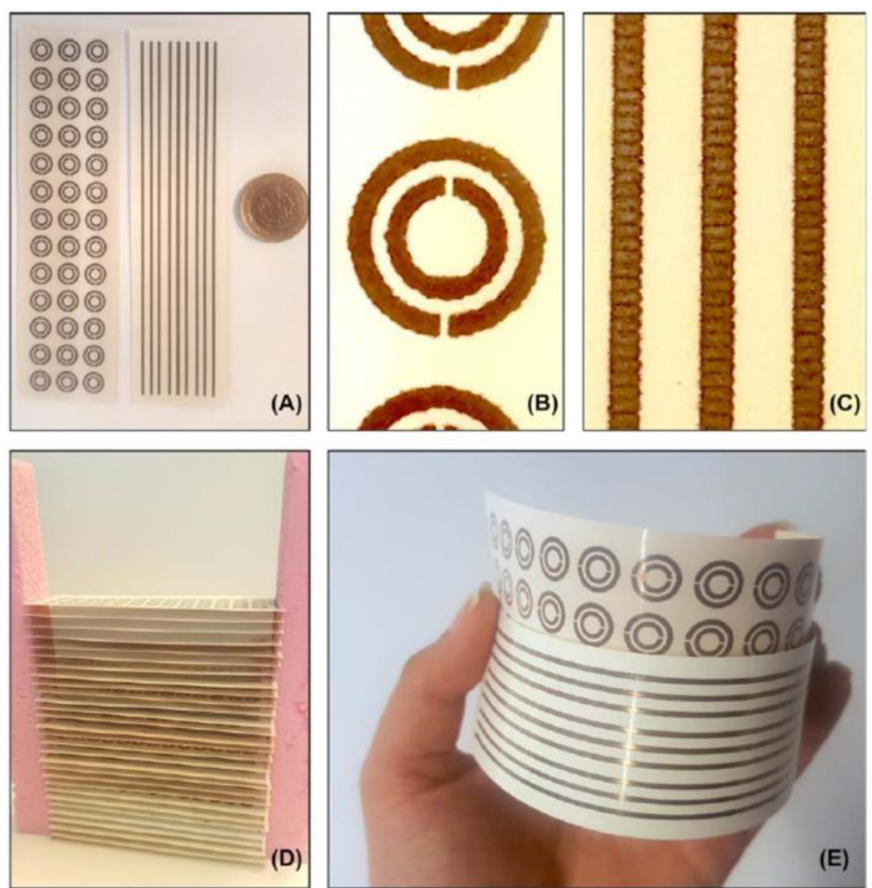

Fig. 2. Various pictures of the fabricated samples, that is, SRRs and thin wires, as well as their combination to construct a composite metamaterial [62]. Reproduced with permission from H. İbili et al., Microw. Opt. Technol Lett. 60, 187 (2018). Copyright 2018 Wiley. (This figure is subject to copyright protection and is not covered by a Creative Commons Commons license.)

İbili et al. demonstrate a kind of inkjet-printed metamaterials which operate at X-band by using silverbased inks, shown in Figure 2 [62]. The SRRs arrays was printed on Canon GP-501 Glossy paper by using a commercial ink-jet printer (Epson Stylus C88+). Then the printed paper was rolled into cylinder to build a 3D metamaterial. The major advantage of this process is that the fabrication cost can be controlled less than 1 USD, which shows great potential for the massive production. 

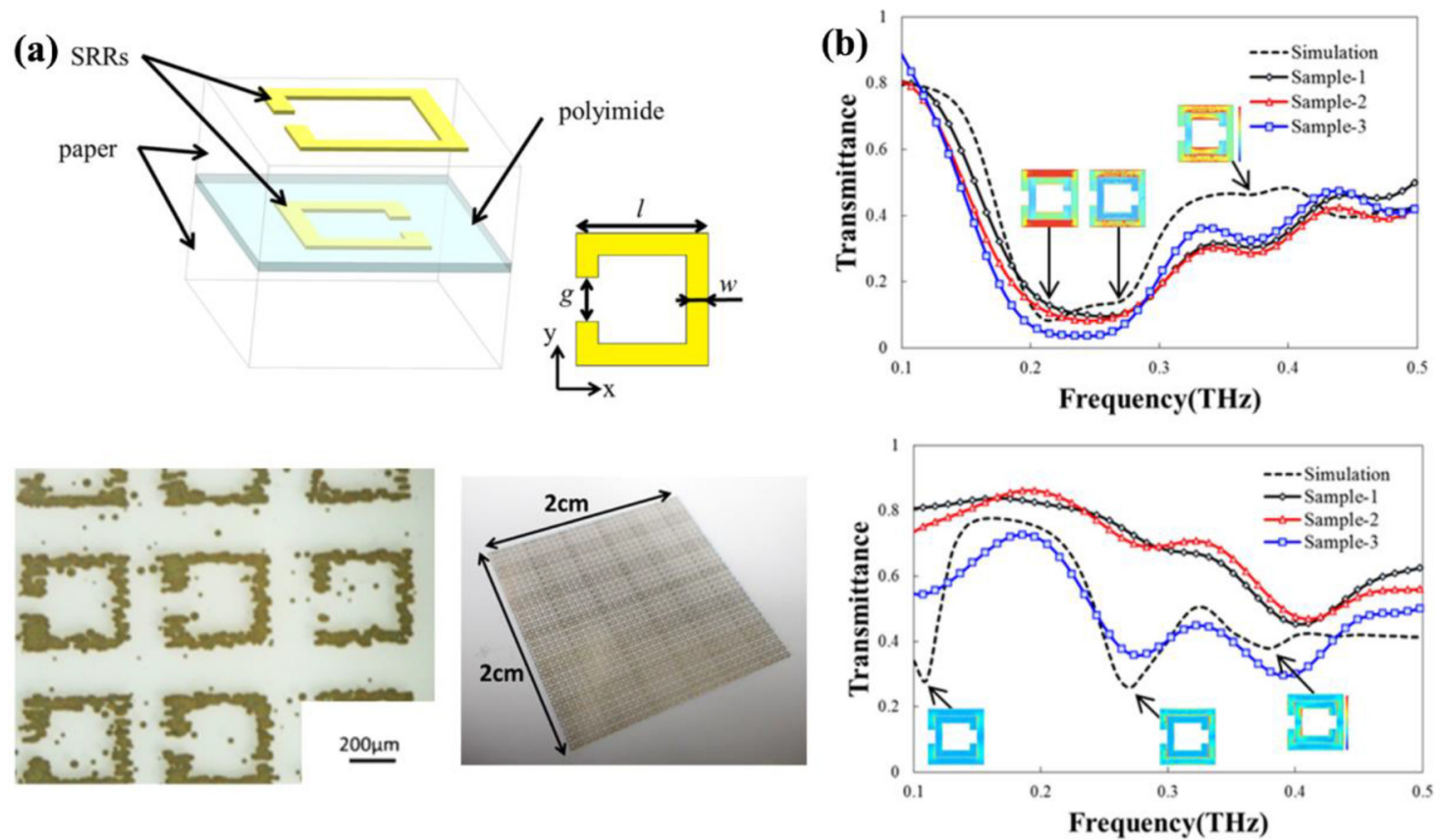

Fig. 3. (a) Schematic diagram and photograph of the paper-based metamaterials. (b) Transmittances of the stacked SRR arrays for $\mathrm{x}$ - and y-polarized incident waves [63]. Reproduced with permission from K. Kashiwagi et al., AIP Adv. 8, 045104 (2018). Licensed under a Creative Commons Attribution (CC BY) license.

Compare to the single-layered paper-based metamaterial, Kashiwagi et al. demonstrate a multiresonant terahertz metamaterials using silver nanoparticle inkjet printing [63]. As shown in Figure 3, two arrays of split-ring resonators (SRRs) with different geometric size have been ink-jet printed on paper respectively. By simply stack them together, the absorption peak of the terahertz metamaterial can be effectively broadened.

It is worth to point out that the ink-jet printing process not only be used in the paper-based metamaterials' fabrication, but also suitable for the other kind of substrate in metamaterials fabrication. Takano et al. using a superfine ink-jet (SIJ) printer to fabricate a terahertz planer metamaterial on Si substrate, which contain a metal wiregrid structure and SRRs arrays (Fig. 4) [64]. The linear resolution of the SIJ printer could reach up to $0.3 \mu \mathrm{m}$, which indicating the SIJ printing method is a powerful tool for terahertz metamaterials fabrication.

The ink-jet printing method has already been widely used in metamaterials fabrication, however, it does have some drawbacks need to be overcome in the near future. Basically, all the studies using ink-jet printing method claim that it is a simple, robust, and cost-effective method for fabricating metamaterial. As for the printing process, it is simple and timesaving. But it needs to be notice that all the printing process require conductive ink (mostly silver ink) as raw material. The ink used for printing process need to be specially designed according to different paper's surface condition, such as surface roughness, porosity. The other issue is that even by using the most advanced ink-jet printer, the resolution can only get to the um level. Thus, the application frequency of the metamaterial fabricated by ink-jet printing process has
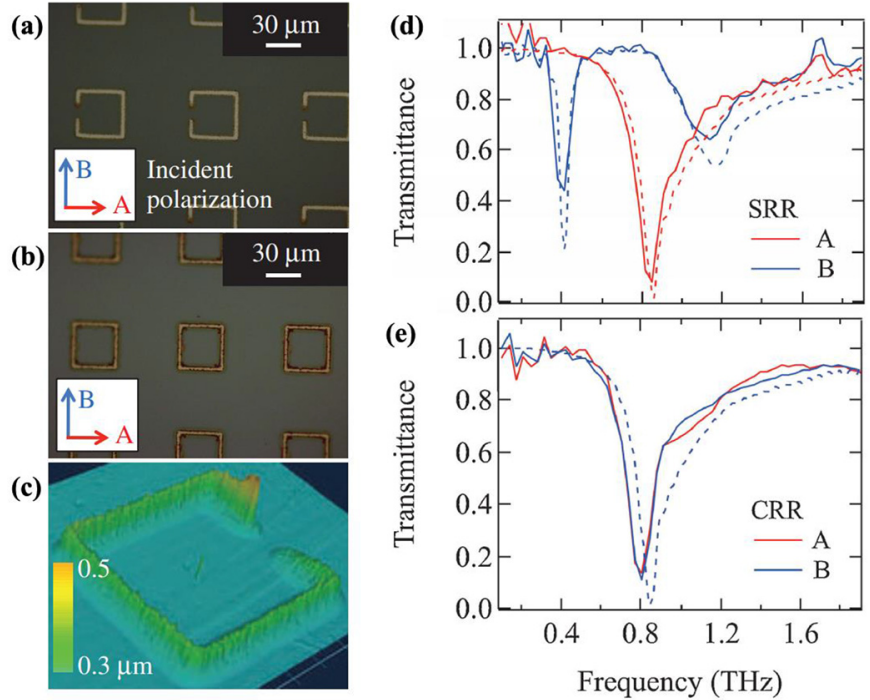

Fig. 4. Microscope photographs of (a) SRRs and (b) CRRs printed on Si substrates, and (c) topographic image of SRRs. Transmission spectra of (d) SRRs and (e) CRRs for incident polarizations [64]. Reproduced with permission from K. Takano et al., Appl. Phys. Express 3, 016701 (2009). Copyright 2009 The Japan Society of Applied Physics. (This figure is subject to copyright protection and is not covered by a Creative Commons Commons license.)

been limited below $\mathrm{THz}$ range due to the resolution limitation of the printer.

\subsection{Direct drawing}

The direct writing is also a convenient, highly efficient and low-cost method for paper-based metamaterial's 
fabrication [65]. In direct drawing process, the conductive trace was direct draw on the substrate for various kinds of applications [66-68].

For example, Tai et al. used direct writing method to construct or repair flexible conductive circuits [69]. Dias et al. used conductive graphite to fabricate electrodes on paper via direct writing process [70].

For the fabrication of paper-based metamaterial, conductive pen is the mostly used tools for the direct writing process. The conductive pen is similar to an ordinary gel pen, except that it contains conductive ink instead of ordinary oil-based ink. The conductive ink is usually made of monodisperse nano-silver colloid, which can offer good conductivity to the pen trace.

Wang et al. drawn split-ring resonators (SRRs) with silver-based conductive ink on papers and constructed the paper-based metamaterials with silver units through this simple pen-on-paper approach for electromagnetic interference shielding purpose (Fig. 5) [71].

Compare to the conductive pen, pencil is another option for the direct writing process.

Pencil is a day-to-day tool which contains graphite and clay particles, sometimes a tiny amount of wax. Depending on the graphite to clay ratio, the conductivity of the pencil trace could be different [72-74], which giving more freedom for properties' design of the paper-based metamaterial $[75,76]$. Hao et al. used both conductive pen and pencil to draw an array of resonant rings on a paper, which shows a broadband microwave absorption property [77].

Compare to the ink-jet printing method, direct writing is a simpler and low-cost method. But in this way, the line width of unit is limited to the diameter of the steel ball on the top of the pen, or the pencil tip. It means that the direct writing method cannot be used for the fabrication of metamaterial for high frequency range application due to the unit size usually require um level precision. Also, the reproducibility of the direct writing process is not as good as the ink-jet printing process.

\subsection{Micro- and nano- fabrication}

In essence, paper is just a kind of flexible substrate. Thus, all the classic micro- and nano- fabrication techniques used for flexible metamaterial fabrication can be used to fabricate paper-based metamaterial, including conventional lithography [78], Focused Ion beam (FIB) [79], shadow mask lithography (SML) [80], electron beam lithography (EBL) [81], laser micro-lens array lithography [82], electroplating [83], and direct laser writing (DLW) [84].

Tao et al. proposed a paper-based metamaterial device to be used for quantitative analysis in biochemical sensing applications [85]. Metallic resonators were deposited on paper with photoresist-free shadow mask deposition technique. Sadeqi et al. used a wax printer to print the substrate on chromatographic paper, then glued a polyimide film with an array of holes, coated a layer of silver ink, and finally peeled off the polyimide sheet (Fig. 6) [86].

Compare to the ink-jet printing or direct writing process in the above section, the micro- and nanofabrication usually contains a series of complicated processes. Although the complicated processes could offer

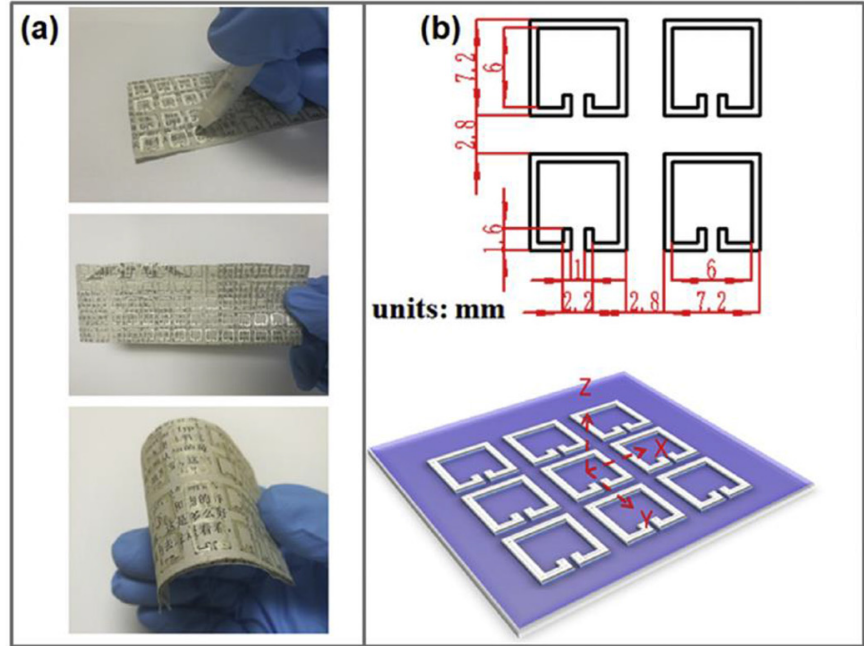

(c)
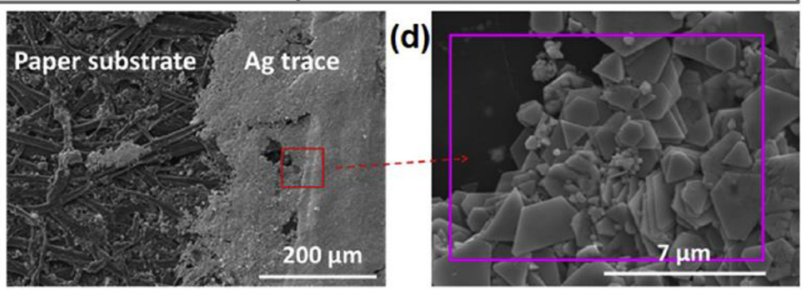

(e)
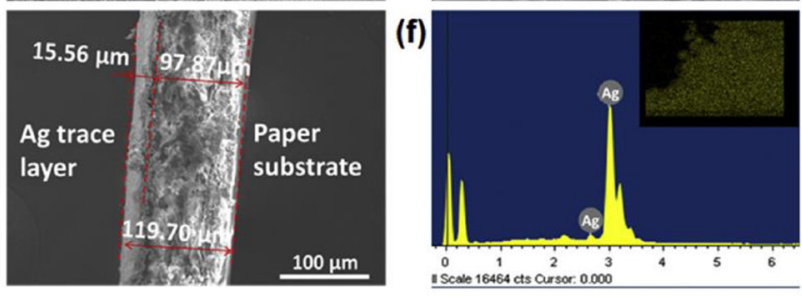

Fig. 5. SRRs designs formed on paper based substrates. (a) SRRs being drawn on paper using rollerball pen with conductive silver ink, (b) Schematic diagram of the electromagnetic simulation model and the dimensions of SRRs, (c and d) SEM images of silver trace on the substrate, (e) SEM image of silver pen trace on a single paper fiber, (f) energy dispersive spectroscopy (EDS) mapping of area marked by square in (d) [71]. Reproduced with permission from Z. Wang et al., J. Clean. Prod. 234, 588 (2019). Copyright 2011 Elsevier. (This figure is subject to copyright protection and is not covered by a Creative Commons Commons license.)

a high precision to the units of the metamaterial, most of them suffer from several difficulties for practical applications, including requirement of high-tech device for fabrication, difficult in mass-production, high fabrication cost, etc. Thus, the micro- and nano- fabrication are limited in laboratory stage and those drawbacks need to be overcome in the future. It is worth mentioning that, among the methods we mentioned in this section, nanoimprint method is a promising method for practical applications, owing to its high precision, low cost, good repeatability and high reliability [87] (Tab. 1).

\subsection{Layer-by-layer stacking to 3D structure}

Most of the paper-based metamaterials are two-dimensional structure, which was also called metasurface. The two-dimensional units design causes several shortcomings, such as anisotropy, polarization sensitivity, and narrow 


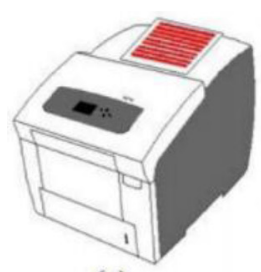

(a)

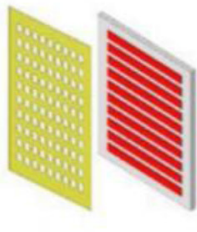

(b)

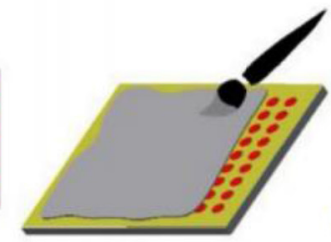

(c)

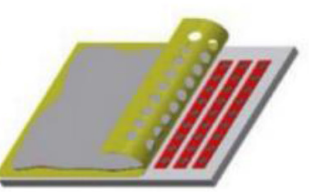

(d)

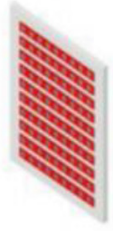

(e)

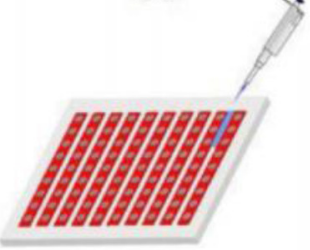

(f)

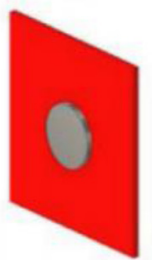

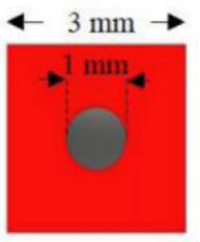

(g)

Fig. 6. Schematic figures showing the Wax printer fabrication process [86]. Reproduced with permission from A. Sadeqi, H.R. Nejad, S. Sonkusale, Opt. Express 25, 16092 (2017). (C) The Optical Society. (This figure is subject to copyright protection and is not covered by a Creative Commons Commons license.)

Table 1. Comparison of the micro- and nano- fabrication methods.

\begin{tabular}{lllllll}
\hline Method & Time consuming & Precision & Price & Area & Unit shape & References \\
\hline EBL & Yes & High & High & Limited & Free & {$[81]$} \\
Mask deposition & No & Moderate & Moderate & Large & Free & {$[85]$} \\
DLW & No & Low & Moderate & Limited & Limited & {$[84]$} \\
nanoimprint & No & High & Low & Large & Free & {$[87]$} \\
FIB & No & High & High & Limited & Free & {$[79]$} \\
Chemistry method & Yes & Low & Low & Large & Limited & - \\
\hline
\end{tabular}

operating bandwidth. Thus, the three-dimensional metamaterial has been paid more and more attention in recent years to overcome the above drawbacks in two-dimensional structure [88-90].

It should be noticed that paper is a typical twodimensional material. It is quite challenging to use paper to fulfill three-dimensional design. In 2020, Fu et al. firstly propose the conception of 2.5D paper-based metamaterial, which provide a new way for the fabrication of threedimensional metamaterial with complex unit structure via two-dimensional patterns on paper [91]. As shown in Figure 7, The procedure is to glue different shapes of metal foil to a paper substrate and then stacking them together. In this way, the trapezoidal prism units (3D structure) can be easily built up. It is worth to point out that any kind of $3 \mathrm{D}$ structure with complex geometrical shape can be easily built up via this staking process by controlling the $2 \mathrm{D}$ shape in each layer. The stacked paper substrates not only exhibit flexibility but also provide strong mechanical strength to the whole structure.

\section{Applications of paper-based metamaterial}

\subsection{Sensor}

Much attention has been paid to the development of practical, cost-effective, easily manufactured diagnostic devices or versatile sensors for environmental monitoring, materials characterization, and even health care [92-95]. (a)
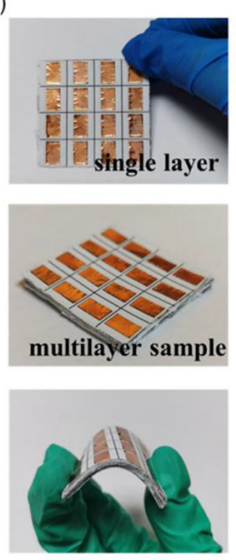

(b)

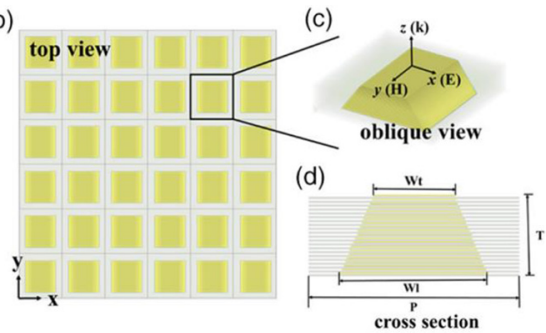

(e)

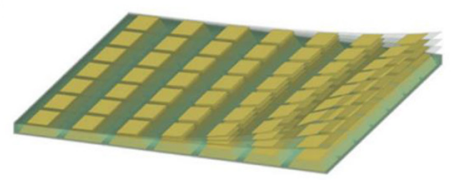

Fig. 7. Design and fabrication of the 2.5D metamaterials: (a) the structural unit was printed on a single layer of paper and stacked together, (b-e) schematic of the 2.5D design: (b) top view, (c) oblique view, (d) 2D cross-sectional view of the structure, and (e) $3 \mathrm{D}$ illustration of the simulated metamaterials [91]. Reproduced with permission from X. Fu et al., Adv. Eng. Mater. 22, 1901126 (2020). Copyright 2020 Wiley. (This figure is subject to copyright protection and is not covered by a Creative Commons Commons license.)

As well known, electromagnetic performance of metamaterials is related to the periodic building blocks and the substrate materials. Based on this, many investigations on 

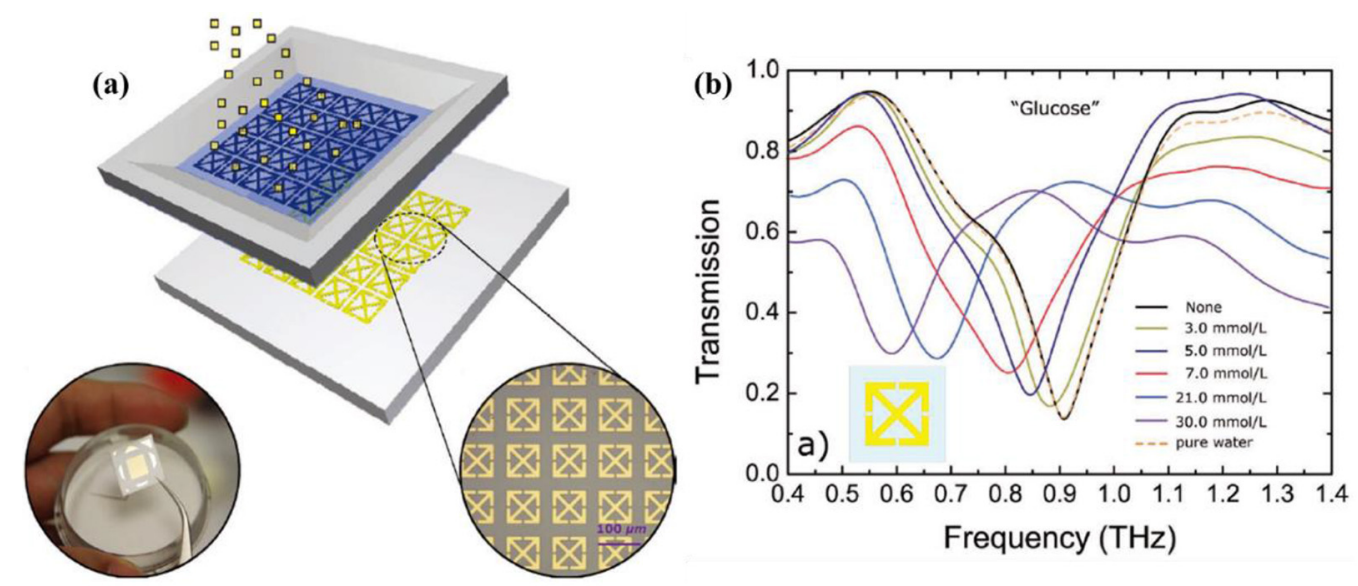

Fig. 8. (a) Schematic diagram and photograph of the paper-based metamaterials. (b) The measured transmission spectra of paperbased metamaterials coated with a series of glucose solutions with different concentration [85]. Reproduced with permission from H. Tao et al., Adv. Mater. 23, 3197 (2011). Copyright 2011 Wiley. (This figure is subject to copyright protection and is not covered by a Creative Commons Commons license.)

paper-based metamaterials have been carried out to seek for an effective sensing platform [96-99]. Hu et al. proposed a paper-based metamaterial device to be used for quantitative analysis in biochemical sensing applications (Fig. 8) [85]. Metallic resonators were deposited on paper with photoresist-free shadow mask deposition technique. Electromagnetic response of the paper-based metamaterials is determined by configuration and size of the resonators. With glucose solutions or other matters are coated on the paper substrate, the resonant responses would be affected. In this case the signature for biochemical sensing applications of the paper-based metamaterials could be detected by monitoring the resonance shift caused by the different materials coated on the paper. In this work, the micrometer-sized metamaterial resonators on paper are responsible for the sensing applications at $\mathrm{THz}$ frequencies. And it's asserted similar performance could be achieved on other regions of the electromagnetic spectrum only by changing the size and configurations of the building block resonators on the paper.

Similarly, Sadeqi et al. fabricated a paper-based metamaterial by the means of wax printing and screen printing [86]. Resonance shift of this kind metamaterials was affected by the effective capacitance of each resonator in the metamaterial arrays, which is essential for the sensing performance. Due to the different dielectric properties of solids like oil, methanol, glycerol, water, and other chemical reagents, the corresponding signature of these solids could be detected on the electromagnetic response of the paper-based metamaterials at $\mathrm{GHz}$ frequencies. In addition, a kind of capacitive wireless RFID-enabled sensing system for capacitive sensing and other Internet of Things applications was proposed, which is sensitive to permittivity of the objective approaching to the on-tag meander line structure of the sensor [100]. The main application of this project includes but not limited to touch sensor, liquid level detection, ambient humidity sensing, and other testing tasks.

\subsection{Broadband microwave absorption}

Microwave absorption of metamaterials has always been a hot topic in past several years [101,102]. Paper-based metamaterials provide a new alternative for broadband microwave absorption owing to lightweight, low cost, easy fabrication, and environment-friendly. Zhao and co-workers drawn resistive strip arrays on paper and intersected the paper sheets to form a vertical square enclosure array structure (Fig. 9) [103].

The results show that this construction can obtain over $90 \%$ absorptivity ranging from 2.52 to $40 \mathrm{GHz}$. The simulation results of the electric field distributions and the surface power loss density distributions suggested that the great power loss was attributed to the loss performance induced at different part of the resistive strip arrays at different frequencies. Meanwhile, strong electric field gathered on the gap between the two unit cells also contributed to the total loss. Thus, the paper-based metamaterials exhibited extremely wideband absorption performance. Thereafter a more facile method that drawing metallic square loops and resistors onto papers was employed to fabricated the paper-based metamaterial absorber [77]. Remarkably the prepared samples achieve $90 \%$ absorptivity as frequency varying from 1.9 to $5.8 \mathrm{GHz}$. Moreover, the wave-absorption performance was dependent on the incident direction of electromagnetic waves. Compared to the metamaterial absorbers composed of metal and dielectric components, the paper-based absorber has advantages in weight, cost, manufacturing and automatization (Tab. 2).

\subsection{Electromagnetic interference shielding}

In recent years, the electromagnetic pollution arose from the widely used wireless communication facilities has stimulated the development of electromagnetic interference shielding materials [108,109]. Much efforts have been made to achieve high shielding effectiveness with paper- 
(a)

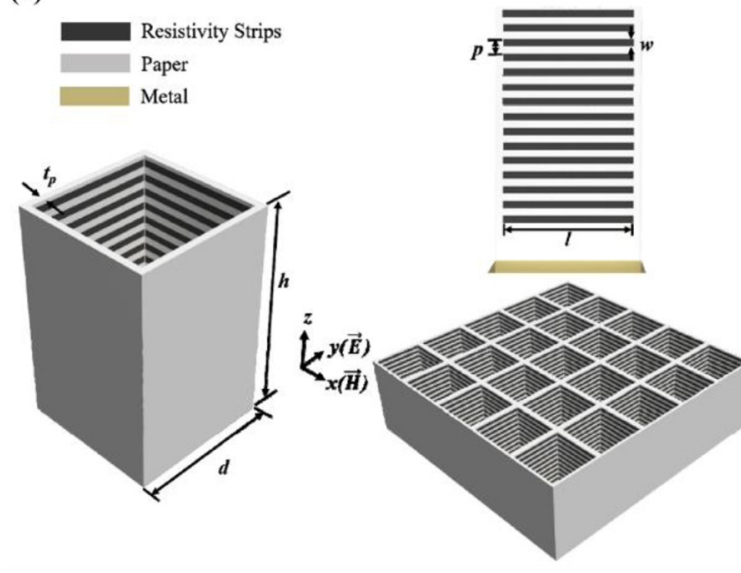

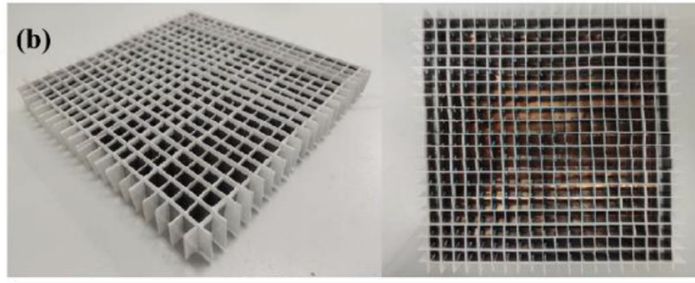

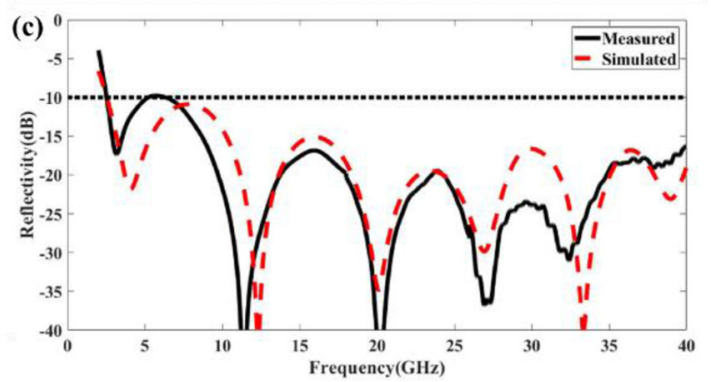

Fig. 9. (a) Schematic of the polarization-independent absorber. (b) Structure of the polarization-independent absorber. (c) Reflectivity of the polarization-independent absorber from the simulation and measurement [103]. Reproduced with permission from Y. Yang et al., 2019 International Symposium on Antennas and Propagation (ISAP), IEEE, 1, 4 (2019). Copyright 2019 IEEE. (This figure is subject to copyright protection and is not covered by a Creative Commons Commons license.)

Table 2. Comparison of microwave absorption properties between paper-based metamaterials and conventional microwave absorption materials.

\begin{tabular}{lllll}
\hline Materials systems & $\begin{array}{l}\text { Preparation } \\
\text { method }\end{array}$ & $\begin{array}{l}\text { Operating } \\
\text { frequency }(\mathrm{GHz})\end{array}$ & $\begin{array}{l}\text { Absorption } \\
\text { bandwidth }(\mathrm{GHz})\end{array}$ & Reference \\
\hline Paper-based metamaterial & Pencil-drawing & $2.52-40$ & 37.48 & {$[103]$} \\
Paper-based metamaterial & Pencil-drawing & $1.9-5.8$ & 3.9 & {$[77]$} \\
$\mathrm{Co} / \mathrm{C}$ nanocomposites & Carbonization & $13.4-17.6$ & 4.3 & {$[104]$} \\
$\mathrm{Fe}_{3} \mathrm{C} / \mathrm{C}$ nanofibers & Electrospinning & $13.3-17.8$ & 4.5 & {$[105]$} \\
$\mathrm{N}-$ doped CNFs & Electrospinning & $12.6-17.5$ & 4.9 & {$[106]$} \\
$\mathrm{Fe} / \mathrm{C}$ nanofibers & Electrospinning & $10.8-13.8$ & 3.0 & {$[107]$} \\
\hline
\end{tabular}

based metamaterials. Wang et al. drawn split-ring resonators (SRRs) with silver-based conductive ink on papers and constructed the paper-based metamaterials with silver units through this simple pen-on-paper approach [71]. Periodic split rings drawn on paper can be regarded as resonator with resonance frequency $f=1 / 2 \pi$ $(L C)^{1 / 2}$, where $L$ is related to the induced currents in SRRs and $C$ is caused by the gaps on SRRs. Thus, based on the coupling resonance of $L C$, the obtained sample exhibited excellent electromagnetic shielding performance.

For the paper-based metamaterial of single-layer, the effective shielding bandwith is almost $3 \mathrm{GHz}$ with the minimum $\mathrm{S}_{21}$ value of $-55 \mathrm{~dB}$. Meanwhile, the frequency band for shielding could be tuned to certain range by changing the orientation relationship of split gap and the electric field component vector. As shown in Figure 10b, the minimum $\mathrm{S}_{21}$ of $-44.78 \mathrm{~dB}$ was observed at $10.4 \mathrm{GHz}$ when the split was pointing downward at $0^{\circ}$, while the minimum $\mathrm{S}_{21}$ of $-38.6 \mathrm{~dB}$ is shifted to $15.02 \mathrm{GHz}$ as the split gap pointed at $90^{\circ}$. Furthermore, effective shielding bandwidth of the paper-based metamaterial of three-layers increased to almost $7 \mathrm{GHz}$ accompanied by the minimum
$\mathrm{S}_{21}$ increased from -55 to $-72 \mathrm{~dB}$, which was attributed to the strong coupling effect induced by adjacent SRRs. Thus, the paper-based metamaterials are promising for higheffective electromagnetic shielding, which not only enriched the applications of metamaterials, but also brought new avenues to recycle waste papers for highperformance devices solving electromagnetic pollution.

\subsection{Antenna}

Another advantage of the paper-based metamaterials is they are flexible, which makes them promising to be used as antenna for wearable communication devices [110,111]. Abdel Aziz et al. printed the antenna with expanded graphite ink on paper substrate [112]. The printed antenna has high electric conductivity of $1 \times 10^{4} \mathrm{~S} /$ square and thin thickness of $30 \mu \mathrm{m}$. Results of the antenna reflection coefficient suggested the antenna has two resonant bands: one is centered at $1.9 \mathrm{GHz}$ and the other is centered at $5.8 \mathrm{GHz}$, of them bandwidth below $-10 \mathrm{~dB}$ is 300 and $400 \mathrm{MHz}$, respectively, as shown in Figure 11b. The normalized simulated radiation pattern measured at 

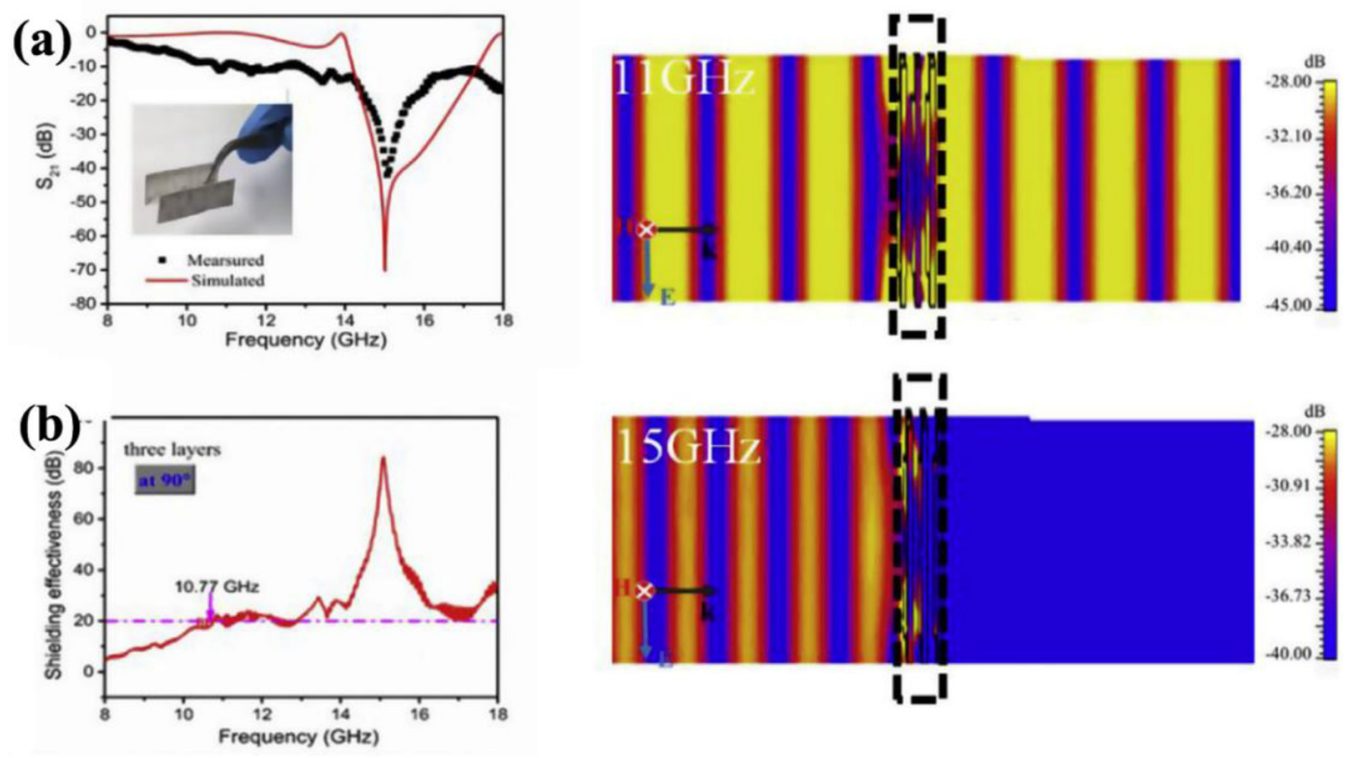

(c)

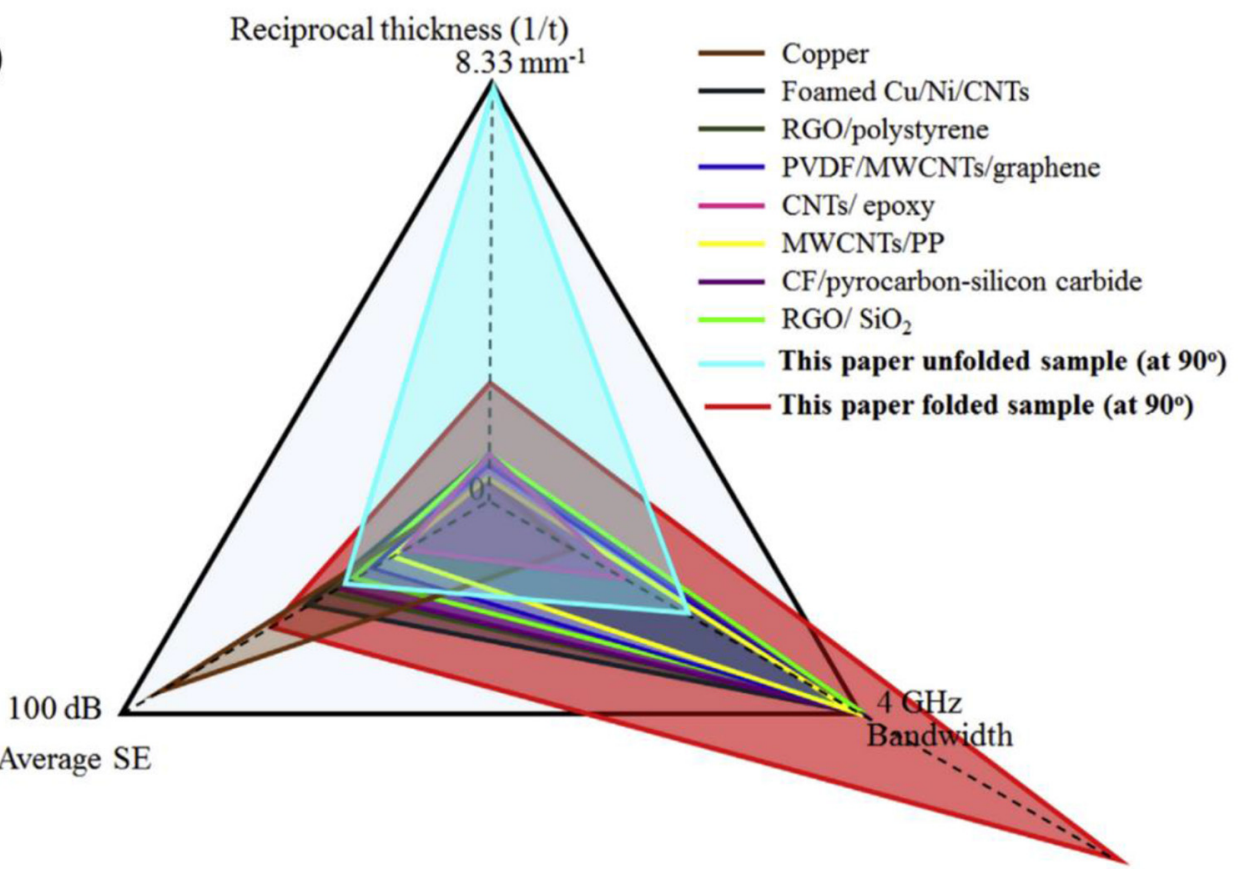

Fig. 10. (a) Experimentally measured and simulated transmission spectra of the three-layer paper-based metasurfaces. (b) The shielding effectiveness of paper-based metasurface in $8-18 \mathrm{GHz}$ (c) The comparison of the SE properties in various materials [71]. Reproduced with permission from Z. Wang et al., J. Clean. Prod. 234, 588 (2019). Copyright 2011 Elsevier.

$1.9 \mathrm{GHz}$ showed that the antenna had a bidirectional pattern in E plane and an isotropic pattern in $\mathrm{H}$ plane, while the pattern measured at $5.8 \mathrm{GHz}$ had also almost bidirectional pattern in E plane and isotropic pattern in $\mathrm{H}$ plane. The small difference between the measured and simulated results may be caused by the flexibility of the proposed antenna. In addition, the measured antenna gain and efficiency at $1.9 \mathrm{GHz}$ is determined to be $-1.266 \mathrm{~dB}$ and $35 \%$, respectively, while that at $5.8 \mathrm{GHz}$ is $0.521 \mathrm{~dB}$ and $55 \%$, respectively. The dual-band printed antenna makes paper-based metamaterials suitable for various wireless applications.

\subsection{Wave filter}

Another application for metamaterials is to filter electromagnetic waves of certain frequencies [113-115]. On the basis of paper-based metamaterials, Zhang et al. proposed the conception of $2.5 \mathrm{D}$ metamaterial, of which the " $3 \mathrm{D}$ " trapezoidal prism units were built up by stacking the rectangle units of $2 \mathrm{D}$ paper-based metasurfaces layer-bylayer [91]. Electromagnetic performance of the 2.5D metamaterials depends on the layers containing metallic patterns, which can be regulated to satisfy impedance matching according to the specific requirements. And the 

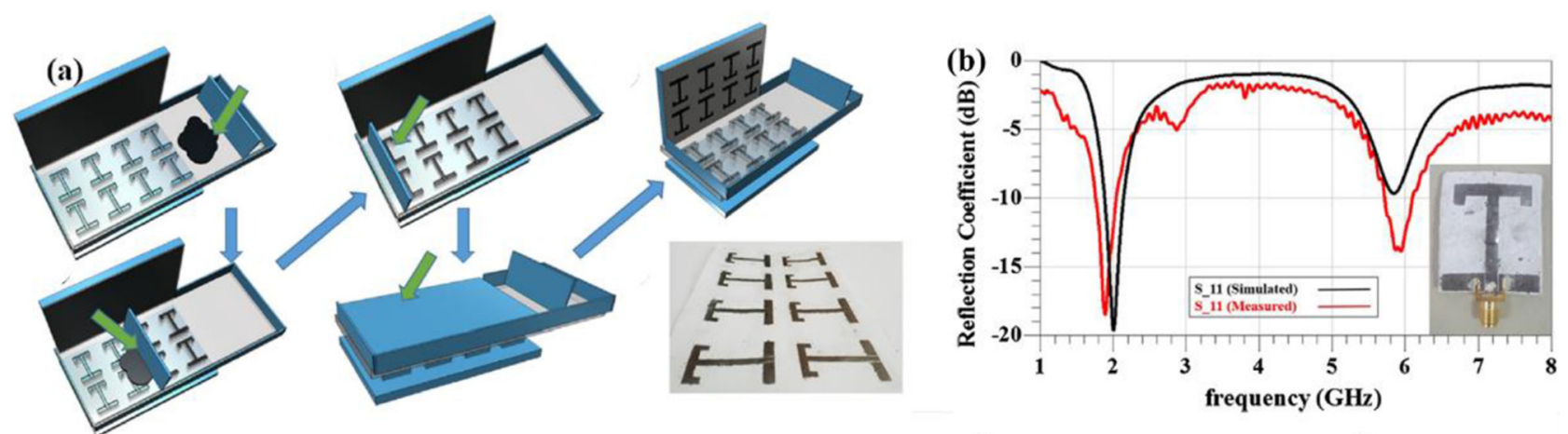

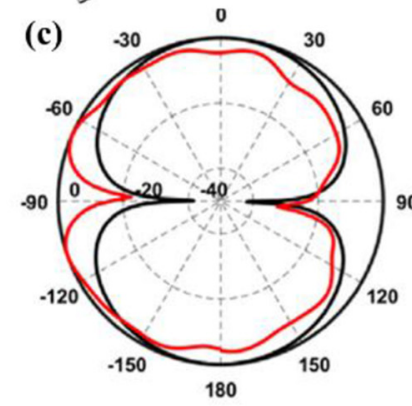

E Plane at $1.9 \mathrm{GHz}$

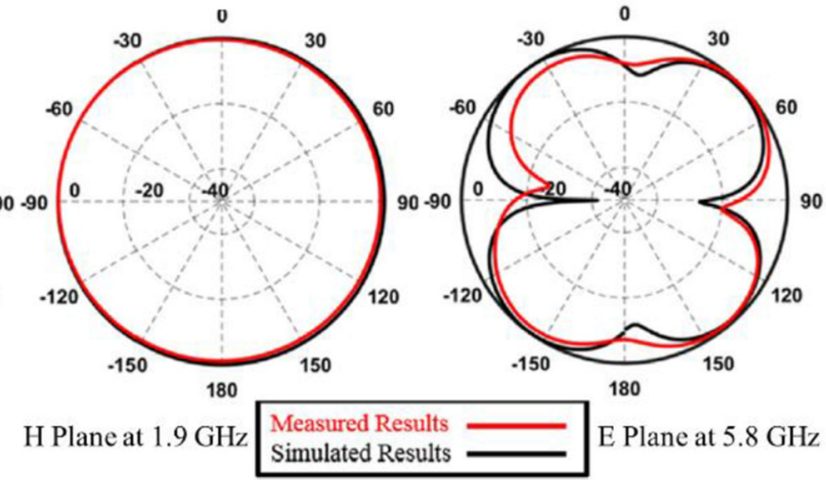

Fig. 11. (a) Schematic diagram for the printing steps of the paper-based antenna. (b) The simulated/measured reflection coefficient of the printed EG antenna. (c) The normalized simulated versus measured radiation pattern in the two planes at 1.9 and $5.8 \mathrm{GHz}$ [112]. Reproduced with permission from A.A. Abdel Aziz et al., Int. J. Electron. Commun. 110, 152869 (2019). Copyright 2019 Elsevier. (This figure is subject to copyright protection and is not covered by a Creative Commons Commons license.)

paper layers of 2.5D metamaterials are used to dissipate the incident waves. Owing to the synergistic effect of these different layers, the 2.5D metamaterials presented shows good wave-filtering performance. The scattering parameter $\mathrm{S}_{21}$ suggested the fabricated $2.5 \mathrm{D}$ metamaterials can be high-efficient wave filters. As shown in Figure 12b, the filtering performance was achieved with $S_{21}$ values lower than $-10 \mathrm{~dB}$ when the frequency was higher than $11 \mathrm{GHz}$, which would effectively prevent incident waves from propagating through the sample. While when frequency was below $11 \mathrm{GHz}, \mathrm{S}_{21}$ was larger than $-10 \mathrm{~dB}$ and that suggested the metamaterials can be considered transparent to the incident waves. Meanwhile, simulation results of electric field distribution also demonstrated the $2.5 \mathrm{D}$ paper-based metamaterials exhibited remarkable filtering performance for the electromagnetic waves near $11 \mathrm{GHz}$. In addition, the interconnected lignocellulosic microfibers in paper endowed the paper-based metamaterials with superior mechanism strength. The great filtering performance was remained even though a pressure loading of $7 \mathrm{MPa}$ was applied on the metamaterial. The conception and demonstration of $2.5 \mathrm{D}$ metamaterial with good wavefiltering property provided a scheme to design the function and structure integrated metamaterials with paper-based metamaterials.

\section{Summary and outlook}

There is no doubt that paper-based metamaterial would play a vital role in the flexible electronic devices due to its extraordinary properties such as low-cost, easy-to-use, recyclable and disposable. Compare to the other flexible metamaterial, the most attractive feature of paper-based metamaterial is that it can be easily folded into different geometrical shape, leading to tunable properties to meet various microwave applications.

As well known, the unit size of the metamaterial is usually on centimeter to millimeter level for microwave application. Basically, all the fabrication method in this review can satisfy the requirement of the size precision. However, to apply the paper-based metamaterial in practice, the fabrication technique needs to be carefully considered. Among all the fabrication techniques mentioned in this review, the ink-jet printing process is a promising method for the mass-production fabrication of paper-based metamaterial due to its high precision, lowcost, time-saving, good reproducibility, as well as the high reliability.

Although the paper-based metamaterial shows great potential in microwave application, there are some drawbacks need to be overcome in the near future. Frist of all, the multi-physics coupling design of metamaterial usually has been ignored. For example, for practical applications, not only the microwave response, but also the mechanical bearing capacity needs to be carefully designed to insure the reliability of the device. However, it usually has been ignored in microwave metamaterial's design, leading to the lacking of the basic structural carrying capacity in paperbased metamaterial. In this case, the paper-based metamaterial will easily be deformed when subjected to external forces, leading to catastrophic failure in maintaining their 

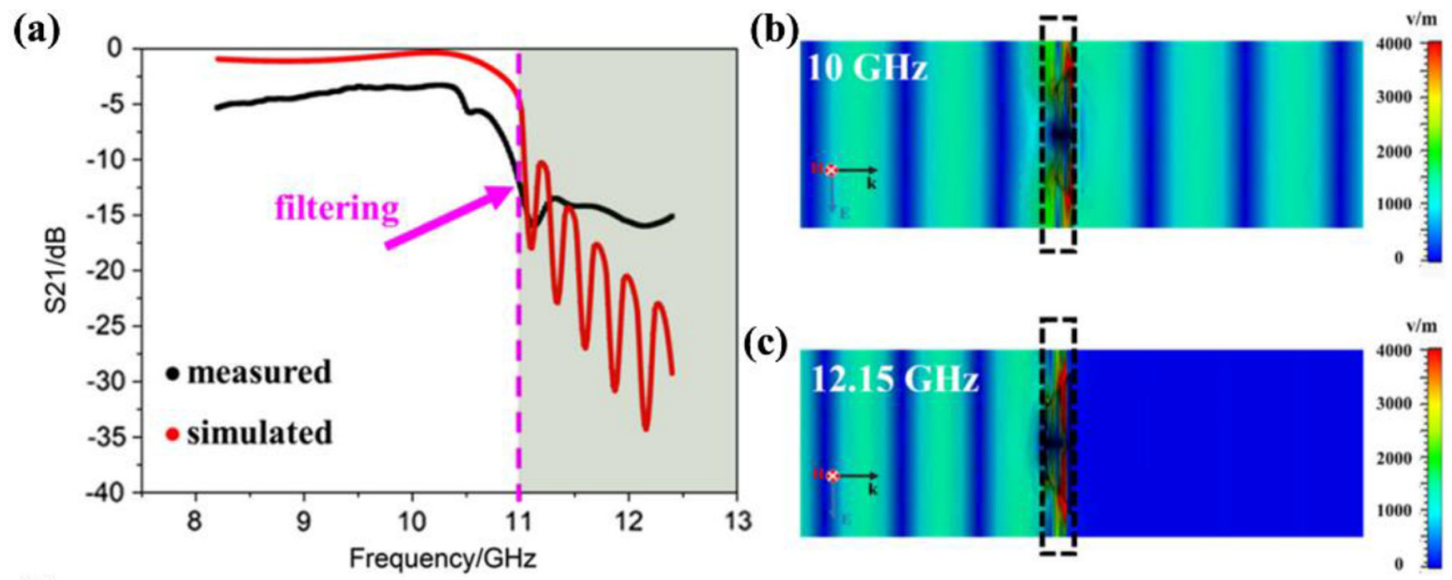

(d)

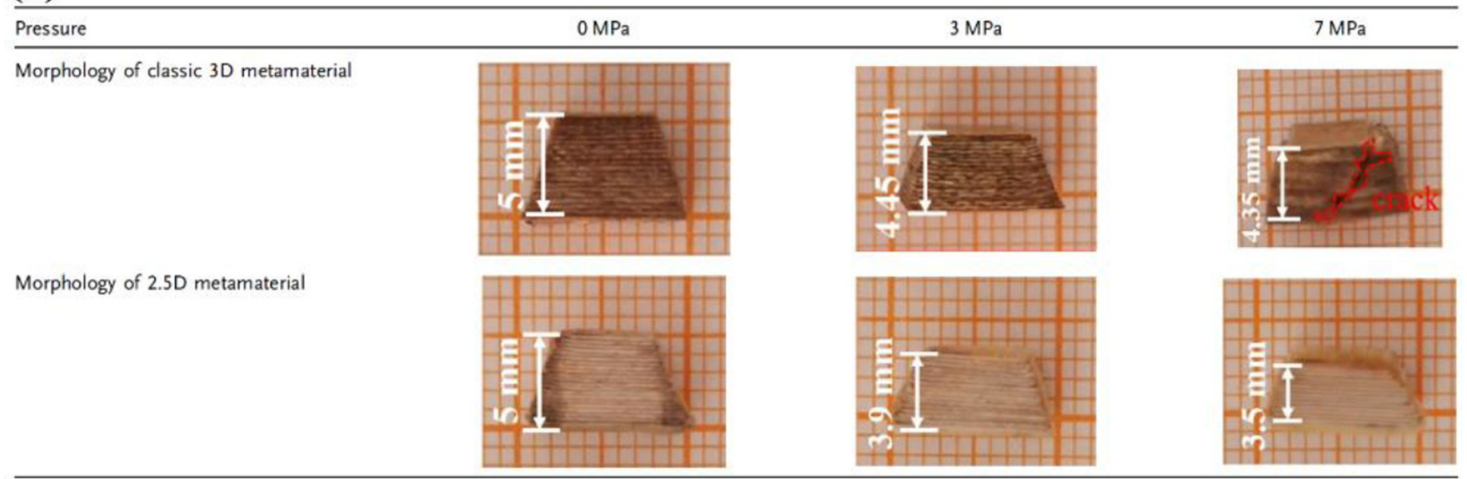

Fig. 12. (a) Simulated and experimental transmission spectra of the $2.5 \mathrm{D}$ paper-based metamaterials. Electric amplitude at (b) 10 and (c) $12.15 \mathrm{GHz}$. (d) The Morphology comparison of 2.5D and 3D metamaterials under different pressures [91]. Reproduced with permission from X. Fu et al., Adv. Eng. Mater. 22, 1901126 (2020). Copyright 2020 Wiley. (This figure is subject to copyright protection and is not covered by a Creative Commons Commons license.)

designed properties. This is unacceptable for the practical applications. Therefore, multi-physics coupling design of paper-based metamaterial, not only including the mechanical property, but also the corrosion resistance, hightemperature resistance, water resistance, etc., needs to be considered in the future research. The other issue is that various kinds of environment parameters could cause irreversible damage to the paper substrate, such as stress, humidness, temperature, etc. Thus, using special paper (e.g. high-temperature resistance paper, waterproof paper.) as the substrate to enhance the mechanical performance of the paper-based metamaterial might be a good idea for the future development of the paper-based metamaterial.

This work was supported by the National Natural Science Foundation of China (Grant No. 51601105, No. 61774020, No. 51871146), China Postdoctoral Science Foundation (Grant No. 2017M622199, 2018T110687), Young Elite Scientists Sponsorship Program by CAST (Grant No. 2017QNRC001), and Innovation Program of Shanghai Municipal Education Commission (Grant No. 2019-01-07-00-10-E00053).

\section{References}

1. J.B. Pendry, Phys. Rev. Lett. 85, 3966 (2000)

2. V.G. Veselago, Sov. Phys. Usp. 10, 509 (1968)
3. N.I. Landy et al., Phys. Rev. Lett. 100, 207402 (2008)

4. P. Xie et al., Research 2019, 1021368 (2019)

5. R.A. Shelby, D.R. Smith, S. Schultz, Science 292, 77 (2001)

6. D.R. Smith, J.B. Pendry, M.C.K. Wiltshire, Science 305, 788 (2004)

7. D. Schurig et al., Science 314, 977 (2006)

8. J. Zhao et al., Nat. Sci. Rev. 6, 231 (2019)

9. S. Narayana, S. Savo, Y. Sato, Appl. Phys. Lett. 102, 201904 (2013)

10. C. Fan, Y. Gao, J. Huang, Appl. Phys. Lett. 92, 251907 (2008)

11. J.H. Lee, J.P. Singer, E.L. Thomas, Adv. Mater. 24, 4782 (2012)

12. X. Zheng et al., Science 344, 1373 (2014)

13. Z. Yang et al., Phys. Rev. Lett. 101, 204301 (2008)

14. H. Chen, C. Chan, Appl. Phys. Lett. 91, 183518 (2007)

15. J. Li, C.T. Chan, Phys. Rev. E 70, 055602 (2004)

16. L. Xin et al., Curr. Opin. Solid State Mater. Sci. 24, 100853 (2020)

17. C. He et al., Phys. Rev. Lett. 123, 195503 (2019)

18. B. Xie et al., Phys. Rev. Lett. 122, 233903 (2019)

19. X. Zhu et al., Phys. Rev. Res. 2, 013280 (2020)

20. J. Valentine et al., Nature 455, 376 (2008)

21. S. Walia et al., Appl. Phys. Rev. 2, 011303 (2015)

22. K. Bertoldi et al., Nat. Rev. Mater. 2, 1 (2017)

23. R. Melik et al., Appl. Phys. Lett. 95, 181105 (2009)

24. A. Di Falco, M. Ploschner, T. F. Krauss, New J. Phys. 12, 113006 (2010) 
25. P.M. Ragi et al., Microw. Opt. Technol. Lett. 54, 1415 (2012)

26. Y. Liu et al., Appl. Opt. 57, 10257 (2018)

27. Q. Zhou et al., Appl. Phys. A 125, 131 (2019)

28. S.M. Kamali et al., Nat. Commun. 7, 1 (2016)

29. M. Choi et al., Nature 470, 369 (2011)

30. S. Aksu et al., Adv. Mater. 23, 4422 (2011)

31. B. Ng et al., Opt. Express 19, 14653 (2011)

32. N.R. Han et al., Opt. Express 19, 6990 (2011)

33. Y. Li et al., Appl. Phys. Lett. 86, 153508 (2005)

34. F. Miyamaru, M.W. Takeda, K. Taima, Appl. Phys. Express 2, 042001 (2009)

35. T. Tumkur et al., Appl. Phys. Lett. 99, 151115 (2011)

36. N. Gibbons et al., Adv. Mater. 21, 3933 (2009)

37. H. Tao et al., Adv. Mater. 23, 3197 (2011)

38. A. Sadeqi, H.R. Nejad, S. Sonkusale, Opt. Express 25, 16092 (2017)

39. K. Ling et al., Opt. Express 23, 110 (2015)

40. S. Wang et al., Biosens. Bioelectron. 31, 212 (2012)

41. S. Vella et al., Anal. Chem. 84, 2883 (2012)

42. L. Ge et al., Anal. Chem. 85, 3961 (2013)

43. M. Liu et al., Angew. Chem. 128, 2759 (2016)

44. J. Ding et al., Angew. Chem. Int. Ed. 55, 13033 (2016)

45. F. Arduini et al., Biosens. Bioelectron. 126, 346 (2019)

46. J.L. Silverberg et al., Science 345, 647 (2014)

47. C. Lv et al., Sci. Rep. 4, 5979 (2014)

48. E. Boatti, N. Vasios, K. Bertoldi, Adv. Mater. 29, 1700360 (2017)

49. K. Fuchi et al., J. Appl. Phys. 111, 084905 (2012)

50. Z. Wang et al., Adv. Mater. 29, 1700412 (2017)

51. Q. He, S. Sun, L. Zhou, Research 2019, 1849272 (2019)

52. T.R. Hebner et al., Appl. Phys. Lett. 72, 519 (1998)

53. H.P. Le, J. Imaging Sci. Technol. 42, 49 (1998)

54. B.K. Park et al., Thin Solid Films 515, 7706 (2007)

55. P.J. Smith et al., J. Mater. Sci. 41, 4153 (2006)

56. R. Hokari et al., Opt. Express 26, 10326 (2018)

57. K. Takano et al., Appl. Phys. Express 3, 016701 (2009)

58. X. Zhu et al., Sci. Adv. 3, e1602487 (2017)

59. A. Paulsen, A. Nahata, 2015 40th International Conference on Infrared, Millimeter, and Terahertz waves (IRMMWTHz), 2015, IEEE, p. 1

60. L.B. Wang et al., IEEE Trans. Electromagn. Compat. 53, 700 (2011)

61. M. Momeni-Nasab et al., Int. J. Electron. Commun. 123, 153259 (2020)

62. H. İbili, B. Karaosmanoglu, Ö. Ergül, Microw. Opt. Technol Lett. 60, 187 (2018)

63. K. Kashiwagi et al., AIP Adv. 8, 045104 (2018)

64. K. Takano et al., Appl. Phys. Express 3, 016701 (2009)

65. N.I. Zheludev, Y.S. Kivshar, Nat. Mater. 11, 917 (2012)

66. A. Russo et al., Adv. Mater. 23, 3426 (2011)

67. L. Polavarapu et al., Small 10, 3065 (2014)

68. Y, Zhang et al., Adv. Mater. 30, 1801588 (2018)

69. Y.L. Tai, Z.G. Yang, J. Mater. Chem. 21, 5938 (2011)

70. A.A. Dias et al., Electroanalysis 30, 2250 (2018)

71. Z. Wang et al., J. Clean. Prod. 234, 588 (2019)
72. S. Cain, A.A. Cantu, R. Brunnelle, A. Lyter, J. Forensic Sci. 23, 643 (1978)

73. J.A. Zoro, R.N. Totty, J. Forensic Sci. 25, 675 (1980)

74. J.A. Denman et al., Forensic Sci. Int. 175, 123 (2008)

75. N. Kurra, G.U. Kulkarni, Lab Chip 13, 2866 (2013)

76. N. Dossi et al., Electrophoresis 34, 2085 (2013)

77. S. Hao et al., 2019 International Conference on Microwave and Millimeter Wave Technology (ICMMT), 2019, IEEE, pp. $1-3$

78. I.E. Khodasevych et al., Appl. Phys. Lett. 100, 061101 (2012)

79. B. Gholipour et al., Adv. Mater. 29, 1604268 (2017)

80. S. Aksu et al., Adv. Mater. 23, 4422 (2011)

81. N. Liu et al., Nat. Mater. 7, 31 (2008)

82. N.R. Han et al., Opt. Express 19, 6990 (2011)

83. K. Fan et al., Phys. Rev. B 87, 161104(R) (2013)

84. T. Buckmann et al., Adv. Mater. 24, 2710 (2012)

85. H. Tao et al., Adv. Mater. 23, 3197 (2011)

86. A. Sadeqi, H.R. Nejad, S. Sonkusale, Opt. Express 25, 16092 (2017)

87. D. Chanda et al., Nat. Nanotechnol. 6, 402 (2011)

88. C.M. Soukoulis, M. Wegener, Nat. Photonics 5, 523 (2011)

89. L. Jing et al., Research 2019, 1 (2019)

90. Q. Zhao et al., Phys. Rev. Lett. 101, 027402 (2008)

91. X. Fu et al., Adv. Eng. Mater. 22, 1901126 (2020)

92. A. Ebrahimi et al., IEEE Sens. J. 14, 1345 (2013)

93. W. Withayachumnankul et al., IEEE Sens. J. 12, 1455 (2011)

94. F. Alves et al., Opt. Lett. 37, 1886 (2012)

95. T.Q. Trung, N.E. Lee, Adv. Mater. 28, 4338 (2016)

96. B.X. Wang et al., J. Appl. Phys. 117, 014504 (2015)

97. T. Chen, S. Li, H. Sun, Sensors 12, 2742 (2012)

98. W. Xu, L. Xie, Y. Ying, Nanoscale 9, 13864 (2017)

99. M. Yoo, H.K. Kim, S. Lim, Sens. Actuators B Chem. 222 $173(2016)$

100. S. Kim et al., 2013 IEEE MTT-S International Microwave Symposium Digest (MTT), 2013, IEEE, pp. 1-4

101. C. Zhang et al., Appl. Phys. Lett. 110, 143511 (2017)

102. J. Zhao et al., Opt. Express 26, 8522 (2018)

103. Y. Yang et al., 2019 International Symposium on Antennas and Propagation (ISAP), 2019, IEEE, pp.1-4

104. G. Fan, et al., J. Alloys Compd. 835, 155345 (2020)

105. Y. Jiang, et al., J. Alloys Compd. 804, 305 (2019)

106. Y. Jiang, et al., J. Mater. Sci. 55, 5832 (2020)

107. F. Wang et al., Carbon 134, 264 (2018)

108. Y. Zhang et al., Opt. Express 28, 26836 (2020)

109. G. Choi et al., Adv. Opt. Mater. 6, 1701076 (2018)

110. J. Xu et al., Research 2019, 9686213 (2019)

111. S. Ghosh, T. N. Tran, T. Le-Ngoc, IEEE Trans. Antennas Propag. 62, 3985 (2014)

112. A.A. Abdel Aziz et al., Int. J. Electron. Commun. 110, 152869 (2019)

113. G. Trainiti et al., Phys. Rev. Lett. 122, 124301 (2019)

114. A. Ali, Z. Hu, IEEE Antennas Wirel. Propag. Lett. 7, 210 (2008)

115. I. Gil et al., 2005 IEEE Antennas and Propagation Society International Symposium, 2005, IEEE, p. 668

Cite this article as: Zidong Zhang, Yaman Zhao, Guohua Fan, Wenjin Zhang, Yao Liu, Jiurong Liu, Runhua Fan, Paper-based flexible metamaterial for microwave applications, EPJ Appl. Metamat. 8, 6 (2021) 